\title{
Murine precision-cut liver slices (PCLS): a new tool for studying tumor microenvironments and cell signaling ex vivo
}

\author{
Alexandra Koch ${ }^{1 \dagger}$, Shashank Saran ${ }^{1 \dagger}$, Doan Duy Hai Tran ${ }^{1}$, Sabine Klebba-Färber ${ }^{1}$, Hauke Thiesler $^{1}$, \\ Katherina Sewald ${ }^{2}$, Susann Schindler ${ }^{2}$, Armin Braun ${ }^{2}$, Robert Klopfleisch ${ }^{3}$ and Teruko Tamura ${ }^{{ }^{*}}$
}

\begin{abstract}
Background: One of the most insidious characteristics of cancer is its spread to and ability to compromise distant organs via the complex process of metastasis. Communication between cancer cells and organ-resident cells via cytokines/chemokines and direct cell-cell contacts are key steps for survival, proliferation and invasion of metastasized cancer cells in organs. Precision-cut liver slices (PCLS) are considered to closely reflect the in vivo situation and are potentially useful for studying the interaction of cancer cells with liver-resident cells as well as being a potentially useful tool for screening anti-cancer reagents. Application of the PCLS technique in the field of cancer research however, has not yet been well developed.
\end{abstract}

Results: We established the mouse PCLS system using perfluorodecalin (PFD) as an artificial oxygen carrier. Using this system we show that the adherence of green fluorescent protein (GFP) labeled MDA-MB-231 (highly invasive) cells to liver tissue in the PCLS was 5-fold greater than that of SK-BR-3 (less invasive) cells. In addition, we generated PCLS from THOC5, a member of transcription/export complex (TREX), knockout (KO) mice. The PCLS still expressed Gapdh or Albumin mRNAs at normal levels, while several chemokine/growth factor or metalloprotease genes, such as Cxc112, Pdgfa, Tgfb, Wnt11, and Mmp1a genes were downregulated more than 2-fold. Interestingly, adhesion of cancer cells to THOC5 KO liver slices was far less (greater than 80\% reduction) than to wild-type liver slices.

Conclusion: Mouse PCLS cultures in the presence of PFD may serve as a useful tool for screening local adherence and invasiveness of individual cancer cells, since single cells can be observed. This method may also prove useful for identification of genes in liver-resident cells that support cancer invasion by using PCLS from transgenic liver.

Keywords: Precision-cut liver slices (PCLS), Perfluorochemicals, Communication between cancer and liver-resident cells, IMARIS image analysis program, THOC5 knockout liver

\section{Background}

The microenvironment of a tumor is one of the essential aspects of tumor growth, progression and invasion. The tumor microenvironment is composed of complex tissues that contain extracellular matrix, activated fibroblasts, immune cells, macrophages, adipocytes, epithelial cells, vascular and lymphatic endothelial cells and a large number of proteins. There are presently two established in vitro and in vivo experimental systems for studying

\footnotetext{
* Correspondence: tamura.teruko@MH-Hannover.de

${ }^{\dagger}$ Equal contributors

'Institut fuer Biochemie, OE4310, Medizinische Hochschule Hannover,

Carl-Neuberg-Str. 1, D-30623 Hannover, Germany

Full list of author information is available at the end of the article
}

metastasis. The first system uses a device called a Boyden or trans-well chamber which constitutes a barrier between two medium-filled compartments allowing cellular invasion to be monitored and quantified. Cells migrate through a porous filter, whereby the filter can be covered with reconstituted extracellular matrix such as Matrigel which mimics a collagen IV-rich basement membrane, fibronectin, laminin, or a fibrillar collagen I-like meshwork [1]. This system, however, cannot predict or mimic the complex interactions between cancer cells and organresident cells (e.g. macrophages, endothelial cells, or fibroblasts) that occur in vivo. The second model that

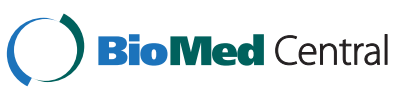

(c) 2014 Koch et al.; licensee BioMed Central Ltd. This is an Open Access article distributed under the terms of the Creative Commons Attribution License (http://creativecommons.org/licenses/by/4.0), which permits unrestricted use, distribution, and reproduction in any medium, provided the original work is properly credited. The Creative Commons Public Domain Dedication waiver (http://creativecommons.org/publicdomain/zero/1.0/) applies to the data made available in this article, unless otherwise stated. 
is used to study native tumor microenvironments is based on several experimental assays in mice that allow emulation of metastatic spread in vivo [2]. Although this is presently the most reliable system this assay fails to measure the earlier invasive stages of malignant progression and does not permit the detailed study of the interaction of cancer cells with organ-resident cells. As a consequence this system may provide an incomplete measure of metastatic capability. Furthermore, this approach requires a large number of experimental animals. As an alternative to the animal model it would be useful to establish a reliable ex vivo approach for the study of malignant metastasis.

Precision-cut liver slices (PCLS) are thought to closely emulate the in vivo situation as they contain all of the cell types present in whole liver while maintaining their three-dimensional structure. Furthermore, the mRNA expression pattern in PCLS is very similar to that in whole liver [3]. A number of slices can be produced from the liver of one animal. This method has been applied to a number of research fields such as toxicology, virology and cancer research [4-10]. Since liver is one of most frequent target organs for metastases of breast cancer, colorectal cancer, esophageal cancer, lung cancer, melanoma, pancreatic cancer, or stomach cancer the PCLS system represents a useful tool for the study of malignant metastasis.

One limitation of this system, however, is the restricted incubation time of viable mouse liver slices. One of the reasons for this restriction may be the high oxygen requirements of liver tissue. Although liver slices were incubated under carbogen $\left(95 \% \mathrm{O}_{2}, 5 \% \mathrm{CO}_{2}\right)$, solubility of oxygen in the culture medium is limited (less than $2.2 \mathrm{mmol} / \mathrm{l}$ ). To address this issue an oxygen carrier is required to extend the life span of the slices. Synthetic, chemically inert, fluorinated compounds called perfluorochemicals are characterized by a carbon chain with all hydrogens replaced by fluorine. One of these chemicals, perfluorodecalin (PFD) possesses an oxygen solubility of $35.5 \mathrm{mmol} / \mathrm{l}$. These oxygen-carrying fluids were originally developed as an alternative to blood for transfusion [11-13], however in vitro applications have only been poorly studied [14-16]. In this report, we describe the modification of the murine PCLS method as an ex vivo system to study tumor microenvironments and cell signaling. We show that co-incubation of mouse PCLS with PFD extended the life span of liver slices. We further show that human cancer cells adhere to the PCLS. Additionally, cancer cells adhered poorly to THOC5 knockout livers $[17,18]$. These data suggest that this method may also prove useful for identification of factors in liver-resident cells that support cancer adherence and invasion by using PCLS from genetically manipulated mice.

\section{Results}

\section{Mouse PCLS culture}

Although the PCLS method using rat livers has been applied to a number of research fields such as toxicology, virology and cancer research $[4-10,19,20]$, murine PCLS has not been widely used. Since a large number of genetically manipulated mice are available, it would be useful to establish a PCLS system for this species. Mouse PCLS were prepared as described in Experimental procedure 1 and Figure $1 \mathrm{~A}$.

\section{Experimental procedure 1: Preparation of murine precision-cut liver slices}

Mice were sacrificed by cervical dislocation. Livers were quickly isolated and transferred into oxygenated ice-cold Krebs-Henseleit buffer (KHB). Preparation of PCLS was mainly performed as described by de Graaf et al. [21] with some modifications (Figure 1). The left liver lobe was separated and placed into a petri dish containing ice-cold KHB. The lobe was cut into pieces of approximately $5 \times 5 \mathrm{~mm}$ using a surgical blade. These pieces were further cut into PCLS of 200-300 $\mu \mathrm{m}$ thickness using a Krumdieck tissue slicer filled with ice-cold KHB using the medium arm speed and blade speed. PCLS were immediately transferred into pre-warmed oxygenated Williams' Medium E (WME) as described in the materials and methods section and were incubated for $3 \mathrm{~h}$ at $37^{\circ} \mathrm{C}$ in a standard cell culture incubator to restore ATP level and wash away cell debris. Finally, PCLS were transferred into fresh WME and were further incubated at $37^{\circ} \mathrm{C}$ under carbogen atmosphere gently shaking the culture plates. WME was exchanged every $24 \mathrm{~h}$. Viability was examined by measuring ATP level extracted from the slices as well as by hematoxylin and eosin (HE)-staining of paraffin sections.

Since the rat PCLS system has been well established $[20,21]$, we first compared the life span of PCLS from mice and rats using two different media, namely WME $[20,21]$, and high glucose Dulbecco's modified eagle medium (DMEM) containing $2 \mathrm{mM}$ sodium pyruvate, $100 \mathrm{nM}$ glucagon, $30 \mathrm{nM}$ insulin, $1 \mu \mathrm{M}$ corticosterone, $1 \mathrm{nM}$ epidermal growth factor (EGF), antibiotics, fungizone, and $5 \%$ heat inactivated and dialyzed fetal calf serum (FCS) [22]. Both rat and mouse slices were prepared as described above and were incubated with these two different media. We first examined the morphology of rat and mouse PCLS. Rat and mouse liver slices that were incubated for 0 or $72 \mathrm{~h}$ were then fixed with formaldehyde, embedded in paraffin and sectioned. The sections were stained with $\mathrm{HE}$ and we counted the nuclei per ten high-power fields (HPFs) from four independent experiments 0 and $72 \mathrm{~h}$ after cutting. In both mouse and rat slices, the numbers of nuclei were reduced to $50 \%$ and $65 \%$ within $72 \mathrm{~h}$, respectively (Figure $2 \mathrm{~A}$ ) in 
A

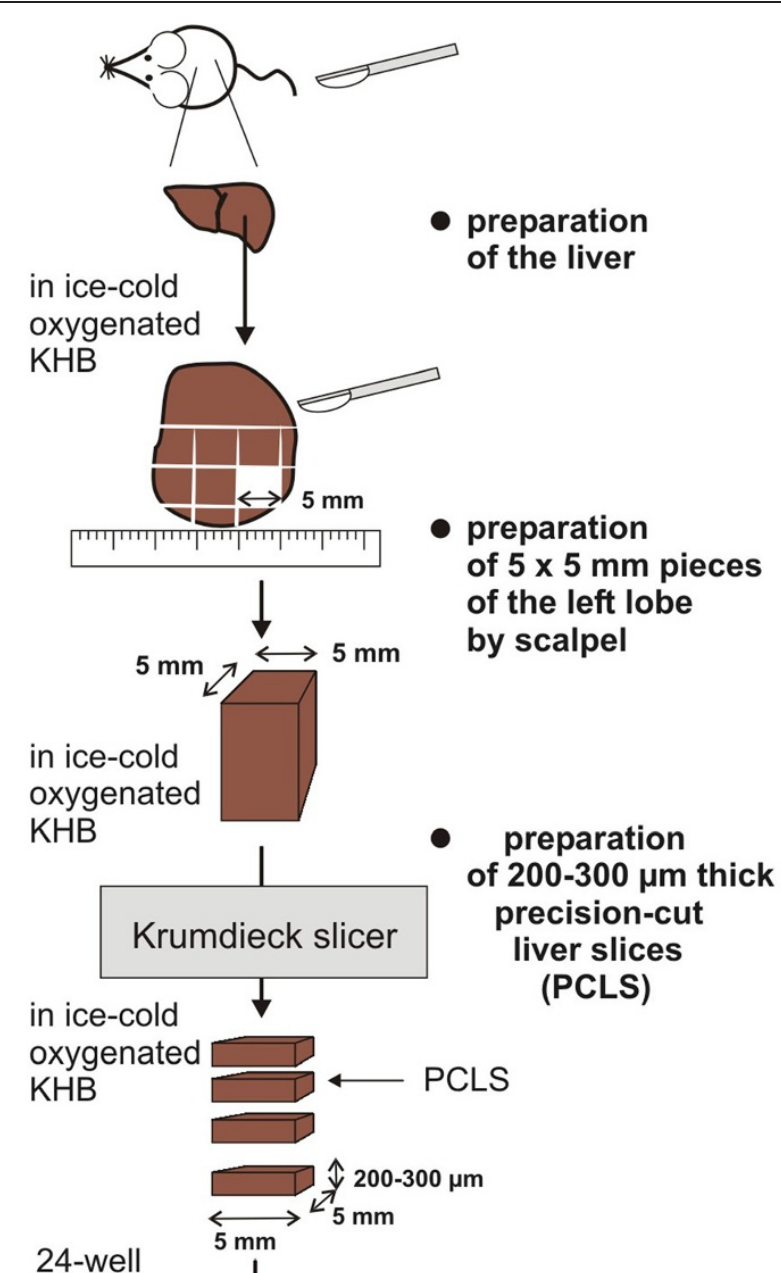

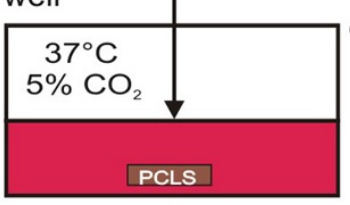

B

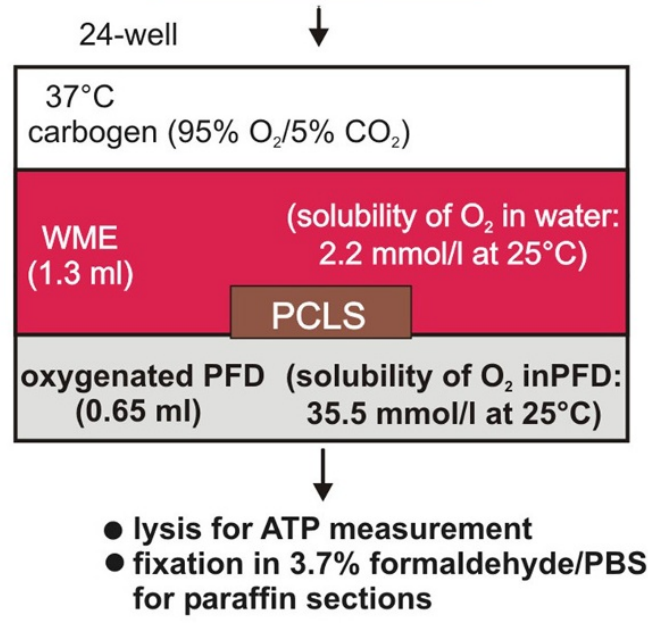

- further culture in a liquid/liquid culture system containing WME (upper phase) and oxygenated PFD (lower phase)

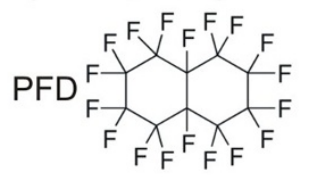

- renew WME and re-oxygenate PFD every $24 \mathrm{~h}$

Figure 1 (See legend on next page.) 
(See figure on previous page.)

Figure 1 Workflow for generation of murine PCLS. (A): Preparation of murine precision-cut liver slices. Mouse livers were transferred into oxygenated ice-cold KHB. The left liver lobe was separated and placed into a petri dish containing ice-cold KHB. The lobe was cut into pieces of approximately $5 \times 5 \mathrm{~mm}$ using a surgical blade. These pieces were further cut into PCLS of 200-300 $\mu$ m thickness using a Krumdieck tissue slicer filled with ice-cold KHB using medium arm speed and blade speed. (B): PFD containing liquid/liquid culture system. After washing, PCLS were transferred into a liquid/liquid culture system. This culture system consists of WME (upper phase) and non-water soluble PFD (lower phase, density: $1.908 \mathrm{~g} / \mathrm{ml}$ at $25^{\circ} \mathrm{C}$ ) that was oxygenated using carbogen $\left(95 \% \mathrm{O}_{2}\right.$ and $\left.5 \% \mathrm{CO}_{2}\right)$. PCLS float on the PFD phase that provides oxygen from the bottom. WME was exchanged, and PFD was re-oxygenated every $24 \mathrm{~h}$. PCLS were cultured up to 3 days in carbogen atmosphere at $37^{\circ} \mathrm{C}$ gently shaking the culture plates.

WME. In DMEM containing growth factors and hormones the number of nuclei in mouse PCLS was less reduced, while that in rat PCLS increased $72 \mathrm{~h}$ after cutting (Figure 2A), suggesting that DMEM containing growth factors and hormones induces hepatocyte proliferation.
Then, ATP level $[20,23]$ in rat or mouse liver slices (6 samples for each from 3 different animals) was measured 0 and $72 \mathrm{~h}$ after cutting. Each value was standardized according to the protein content of each preparation (Figure 2B). In agreement with morphological data, both

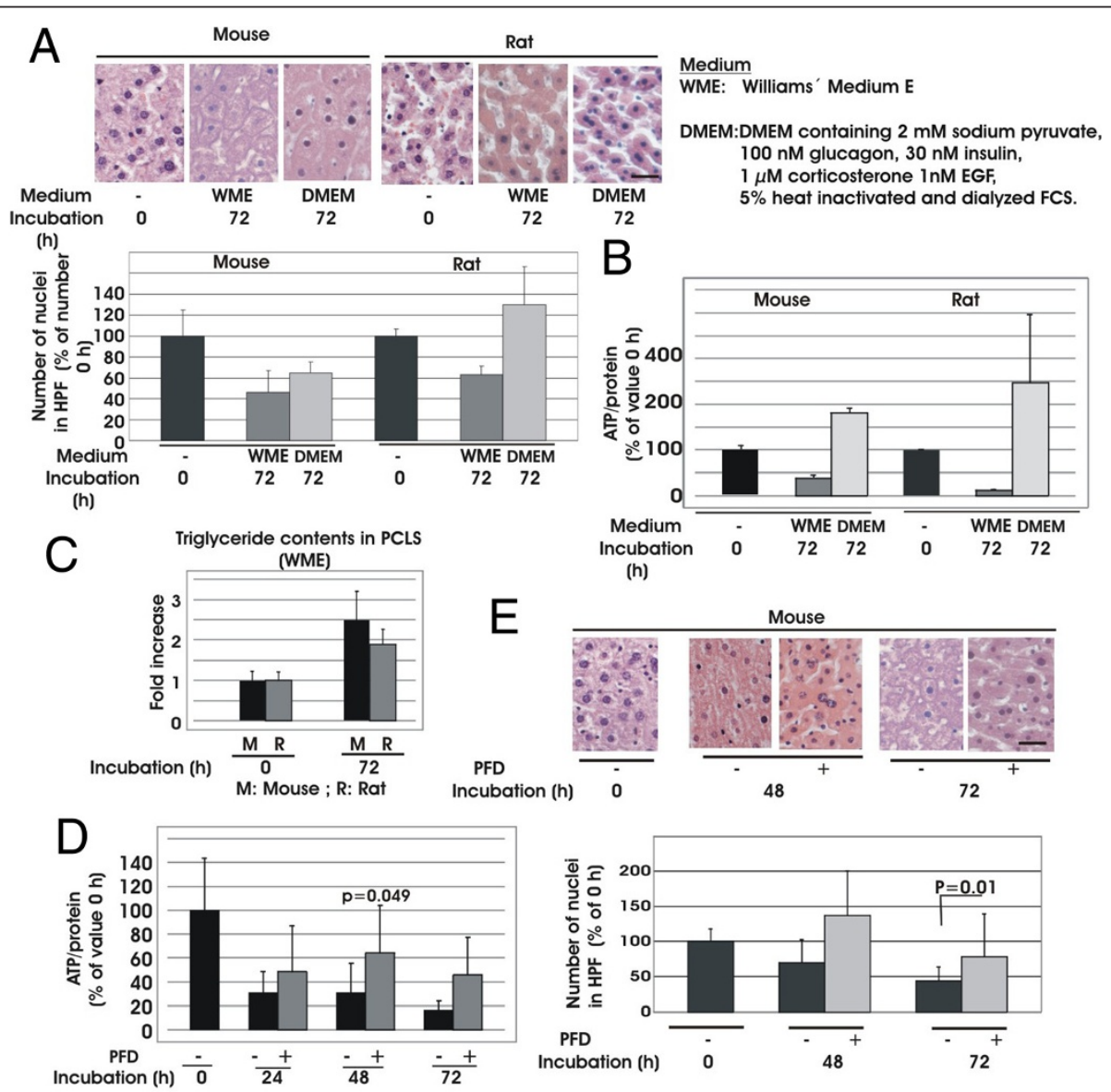

Figure 2 Application of perfluorodecalin for PCLS culture. (A-C): Comparison between mouse and rat PCLS: PCLS were generated from rat or mouse using a Krumdieck tissue slicer as described in Figure 1 and incubated for 0 and $72 \mathrm{~h}$. Three independent experiments (6 slices for each sample) were performed. Slices were incubated either in Williams' medium E (WME) or DMEM containing sodium pyruvate, glucagon, insulin, corticosterone, EGF and 5\% dialyzed FCS. (A): Paraffin sections (5 $\mu \mathrm{m}$ thick) were prepared and stained with HE. An example of representative data 0 and $72 \mathrm{~h}$ after slicing is shown (bar represents $50 \mu \mathrm{m}$ ) and numbers of nuclei in hepatocytes per ten HPF from 4 independent animals (average of number of nuclei before ex vivo incubation as 100\%) are shown. (B): ATP level was measured using ATP determination kit (Biaffin $\mathrm{GmbH).} \mathrm{(C):} \mathrm{Triglyceride} \mathrm{content} \mathrm{in} \mathrm{rat} \mathrm{and} \mathrm{mouse} \mathrm{PCLS} \mathrm{incubated} \mathrm{in} \mathrm{the} \mathrm{WME} \mathrm{medium} \mathrm{for} 72 \mathrm{~h}$ was measured using Triglyceride colorimetric assay kit and fold-increase from value before ex vivo incubation are shown. (D): PFD treatment delayed the loss of ATP levels in the mouse PCLS: PCLS were incubated 0, 24, 48 and $72 \mathrm{~h}$. ATP levels were measured from each slice (12 slices from 4 independent experiments in each condition) and the percentage of ATP is represented (value before ex vivo incubation as 100\%). (E): HE staining of paraffin sections (5 $\mu \mathrm{m}$ thick) obtained from 0, 48 and $72 \mathrm{~h}$ samples with (+) or without (-) PFD. Bar represents $50 \mu \mathrm{m}$. Numbers of nuclei in hepatocytes per ten HPF from 4 independent animals (average value before ex vivo incubation as 100\%). P value: student's $t$-test. 
mouse and rat slices lost more than 60\% ATP level within $72 \mathrm{~h}$ in WME medium, however in DMEM with growth factors and hormones ATP level instead increased within $72 \mathrm{~h}$ (Figure 2B).

To quantify cell demise in these PCLS, we next measured lipid changes by triglyceride assay. In mouse and rat PCLS in WME medium, the level of triglyceride increased 2.5- and 1.8-fold within $72 \mathrm{~h}$ (Figure 2C). These data imply that DMEM with growth factors and hormones clearly prolong the life span of PCLS. However, DMEM contains several supplements such as corticosterone, insulin, glucagon or growth factors. Especially EGF and insulin activate tyrosine kinases which are involved in cancer formation. Over decades, numerous laboratories have studied the link between insulin-like growth factor (IGF), insulin, and growth hormone with the development and progression of breast cancer [24], rendering this medium unsuitable for our application in cancer cell biology Therefore, an alternative method, beside adding growth factors and hormones, is required to prolong viability of mouse PCLS. We modified the protocol for mouse PCLS culture using oxygen supplier, PFD, because high oxygen requirements are known to be an important requirement of liver tissue. Indeed, in humans, the liver is involved in $20-30 \%$ of total oxygen consumption.

\section{Experimental procedure 2: A liquid/liquid culture system containing PFD}

After incubation for $3 \mathrm{~h}$ at $37^{\circ} \mathrm{C}$, PCLS were transferred into a liquid/liquid culture system (Figure 1B). This culture system consists of WME (upper phase) and non-water soluble PFD (lower phase, density: $1.908 \mathrm{~g} / \mathrm{ml}$ at $25^{\circ} \mathrm{C}$ ) that was oxygenated using carbogen $\left(95 \% \mathrm{O}_{2}\right.$ and $\left.5 \% \mathrm{CO}_{2}\right)$. PCLS float on the PFD phase that provides oxygen from the bottom. WME was exchanged, and PFD was reoxygenated every $24 \mathrm{~h}$. PCLS were cultured up to 3 days in carbogen atmosphere at $37^{\circ} \mathrm{C}$ gently shaking the culture plates. Viability was examined by measuring ATP level extracted from the slices as well as by HE-staining of paraffin sections (Figure 1B).

Mouse PCLS were prepared as described in Experimental procedure 1 and were incubated in the presence or absence of PFD. We first examined the ATP levels in PCLS (12 samples for each from 4 independent experiments) $0,24,48$, and $72 \mathrm{~h}$ after cutting. As shown in Figure 2D, ATP level was reduced to $30 \%$ in the absence of PFD within $48 \mathrm{~h}$, while in the presence of PFD PCLS retain more than $65 \%$ of the original ATP level at the same time point, indicating that the PFD treatment has a dramatic effect on PCLS survival in vitro. We also examined cell damage using the triglyceride assay, however the triglyceride level increased 2.4-fold in both PFD treated and untreated PCLS (data not shown).
We then examined the morphology of PCLS. Liver slices that were incubated for 0,48 or $72 \mathrm{~h}$ in the presence or absence of PFD, were fixed with formaldehyde, and paraffin sections were prepared. The sections were stained with HE. As shown in Figure 2E, in the absence of PFD, the hepatocytes all became necrotic within $72 \mathrm{~h}$, whereas in the presence of PFD, the number of necrotic hepatocytes was greatly reduced. We counted the number of hepatocyte nuclei per ten HPFs from four independent experiments. In the presence of PFD, the number of hepatocytes increased within $48 \mathrm{~h}$ in PCLS, while in the absence of PFD hepatocytes decreased 50\% (Figure 2E). These data suggest that an oxygen carrier, PFD, increases the life span of PCLS and/or maintains regenerative signals.

\section{Adhesion assay of cancer cells to PCLS}

The question arises as to whether PFD treated PCLS cultures may serve as a useful tool for physiologically relevant preclinical oncology studies. Since PCLS contain a native extracellular matrix, cytokines/growth factors and liver-resident cells that interact with cancer cells, such as Kupffer cells or fibroblasts, they may serve as a suitable model for studies of tumor microenvironment. After extravasation of tumor cells into parenchyma of liver, cells initially survive in these microenvironments and are able to form micrometastases. Tumor cells then reinitiate their proliferative program at the liver for metastatic colonization [25]. We examined whether the PCLS method can be applied to the study of survival of cancer cells in microenvironments of distant tissue. We chose two breast cancer cell lines, namely MDA-MB231 and SK-BR-3 cells, because both were isolated from pleural effusion but not from liver. Notably, MDA-MB231 cells have been reported to migrate 10-fold further than SK-BR-3 cells using the Boyden chamber in vitro system [26,27]. The adhesion assay using PCLS with cancer cells is described in Experimental procedure 3 and Figure 3A. Since ATP levels were reduced between 48 and $72 \mathrm{~h}$ incubation time (Figure 2D), the experiments with cancer cells were conducted during $48 \mathrm{~h}$ of incubation.

\section{Experimental procedure 3: Cancer cell adhesion assay}

MDA-MB-231 or SK-BR-3 cells were infected with adenovirus carrying green fluorescent protein (GFP) (multiplicity of infection: 50$) 24 \mathrm{~h}$ prior to seeding onto the PCLS (Figure 3A). More than 95\% of the cells expressed GFP in all cases (Figure $3 \mathrm{~B}$ ). Cancer cells were washed thrice with PBS and detached from the culture dish using 0.02\% EDTA in PBS for 15 min. EDTA was removed by centrifugation. The cells were then suspended in WME containing $2 \%$ FCS and $1 \times 10^{5}$ cells were seeded per well of a 24 well plate. Cancer cells were equally distributed within the well 


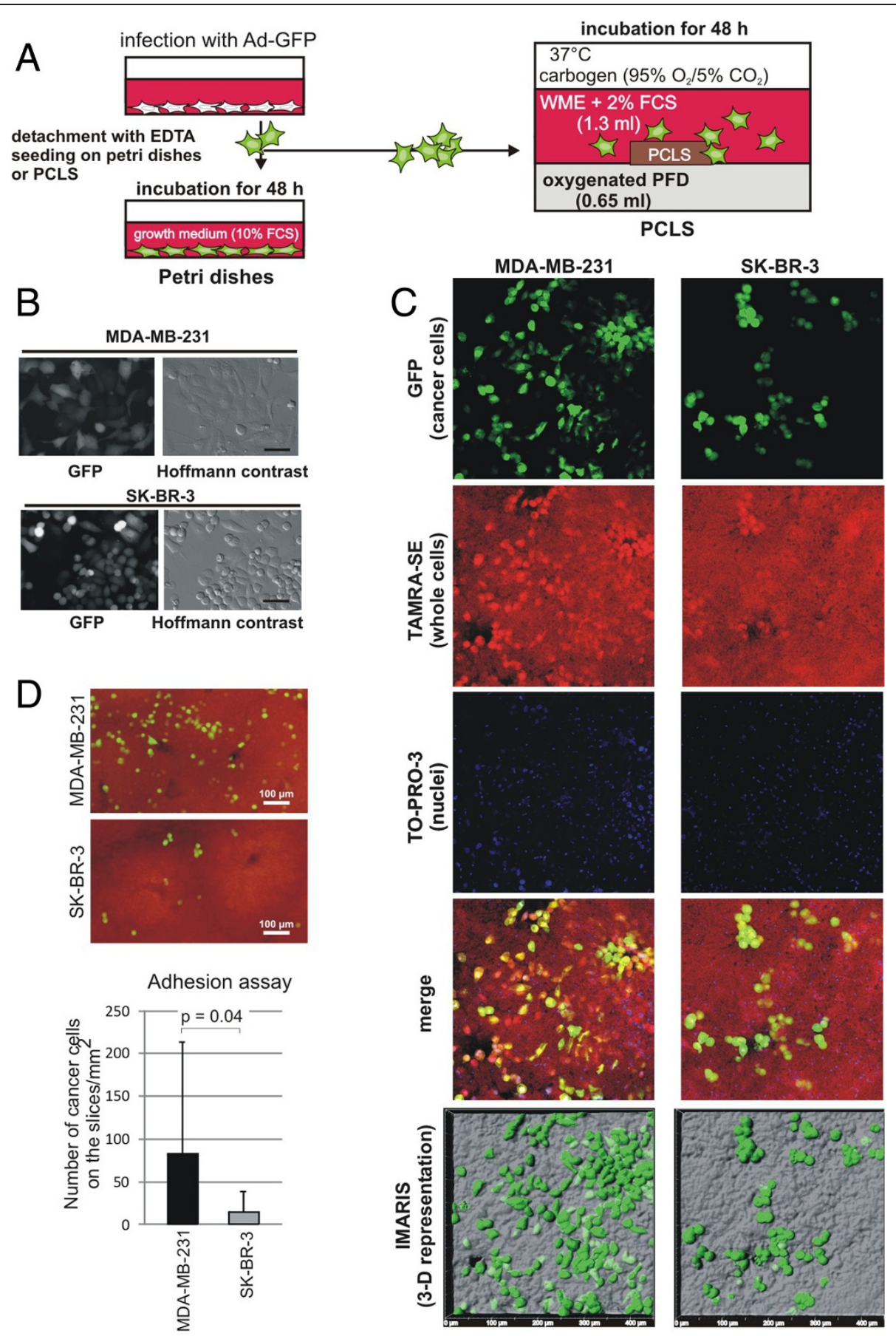

Figure 3 Cancer cells adhere onto liver slices. (A): Workflow for the cancer cell adhesion assay using murine PCLS. Cancer cells, MDA-MB-231 or SK-BR-3 cells, were infected with adenovirus carrying GFP (Ad-GFP) 24 h prior to seeding onto petri dishes or PCLS. Cells on the petri dish were incubated in their normal growth medium. For the cancer cell adhesion assay, $1 \times 10^{5}$ cells were suspended in WME containing $2 \%$ FCS and were plated onto PCLS in a PFD-containing liquid/liquid culture. Cells were further incubated for 48 h. (B): Living cells on the petri dish were photographed using a Nikon Eclipse TE 300 fluorescence microscope (Düsseldorf, Germany). Bar represents 30 m. (C): PCLS with cancer cells (green) were fixed, counterstained by TAMRA (red; whole cells) and TO-PRO-3 (nuclear staining; blue in this figure) and photographed using a confocal microscope. merge: Merged photos with three colors. IMARIS (3-D representation): IMARIS analysis software was applied to reconstruct 3-D images. The tissue surface of PCLS (half transparent grey) with cancer cells (green) is shown in top view. (D): Quantitative determination of number of adherent cancer cells: PCLS (red) with MDA-MB-231 (green) or SK-BR-3 cells (green) photographed with a Nikon Eclipse TE 300 fluorescence microscope. GFP positive cells were counted from 18 slices for each cell line from three different mice. P value: student's t-test. 
by gently pipetting the WME phase up and down three times. PCLS and cancer cells were co-incubated in WME containing 2\% FCS without shaking to allow adherence for $48 \mathrm{~h}$. Medium was renewed and PFD was re-oxygenized every $24 \mathrm{~h}$. Finally, PCLS were fixed in PBS-buffered $3.7 \%$ formaldehyde for further analysis by fluorescence microscopy or immunohistochemistry. After counter staining, fixed liver slices were examined by confocal microscopy. High resolution, three-dimensional (3-D) reconstruction of GFP positive cancer cells in PCLS was performed using the IMARIS image analysis software. Quantification of cell adhesion: Formaldehyde fixed PCLS were mounted on coverslips using Mowiol containing DABCO (1,4-diazabicyclo-[2.2.2] octane, Sigma-Aldrich Chemie GmbH, Steinheim, Germany) (100 mg/ml). Fluorescence images of PCLS were photographed using a $4 \times$ objective of a Nikon Eclipse TE300 microscope to show GFP-expressing cancer cells. Background fluorescence of the liver tissue was utilized to reconstruct the whole PCLS. Finally, GFP-containing cells were counted from the whole PCLS-area. The cell number per $\mathrm{mm}^{2}$ was determined.

In vivo cancer metastasis generally occurs via blood or lymphatic vessels however, in this experiment we plated cancer cells on liver slices. To determine whether cancer cells migrated to particular regions in the liver slices, such as liver plates, central vein or branches of the hepatic artery, we further examined these slices. PCLS with cancer cells (GFP positive) were stained by TAMRA-SE (for whole cells) and TO-PRO-3 iodide (nuclear staining) and analyzed by confocal microscope (Figure $3 \mathrm{C}$ ). Most GFP positive adhered cells were observed on the liver plates. MDA-MB-231 cells were found distributed throughout the slices, while SK-BR-3 cells adhered and had a more spherical shape (Figure $3 \mathrm{C}$ ).

To further quantify the adhesion potential of cells to PCLS, we counted MDA-MB-231 and SK-BR-3 cells on the PCLS (Figure 3D). Strikingly, the adherence of MDA-MB-231 cells to liver tissue in the PCLS was 5 -fold greater than that of SK-BR-3 cells (Experiments were performed three times and GFP positive cells were counted on 18 slices for each cell line). Difference in adhesion between the two cell lines may be due to the difference in substances on the cancer cell surfaces that adhere to liver resident cells. Since slicing by itself is traumatic to tissue, this may induce cellular signaling such as apoptosis signaling soon after cutting. To examine whether tissue damage plays a key role in cancer cell adhesion, we incubated liver slices for $24 \mathrm{~h}$ for recovering from the damage and then added MDAMB-231 cells. We did not find any significant difference in adhesion potential of slices to cancer cells between $3 \mathrm{~h}$ or $24 \mathrm{~h}$ after slicing (data not shown). In order to examine the effect of fungizone on adhesion [28], we performed cancer cell adhesion assay without fungizone. Adhesion of MDA-MB-231 cells to PCLS was not different in the presence or absence of fungizone (data not shown).

To examine whether the PCLS system is suitable for observation of single cells, we analyzed the 3-D images using a confocal microscope followed by IMARIS analysis. We observed that single MDA-MB-231 cells can be detected 25 to $27 \mu \mathrm{m}$ beneath the surface of the slices and single SK-BR-3 cell was observed to a depth of about $16 \mu \mathrm{m}$ (Figure 4). In both cases invadopodia like structures [29] were observed. These data suggest that this method is also suitable for direct visualization and analysis of tumor behavior at single-cell resolution ex vivo. However, since very few cells penetrated the slices, it is not clear whether this is from an early stage of micrometastasis formation or whether this is due to the rough and irregular surface after the cutting which may lead to cuts/scars in which the cancer cells are more capable of invasion. To further study further proliferation and invasion, we need to optimize the mouse PCLS culture conditions and further prolong the life span of the PCLS. In any case, however, the PCLS method is suitable to study the effect of exogenous factors for cancer cell adhesion in tissue.

\section{Application of PCLS derived from genetically manipulated mice for studying tumor microenvironments and cell signaling at the molecular level}

We next examined whether PCLS can be applied as a model system to study the communication between cancer cells and organ resident cells at the molecular level. We have recently identified a potential target molecule for cancer therapy, THOC5, a member of the mRNA export complex [30]. THOC5 is a nuclear protein and is responsible for the processing of only approximately 100 mRNAs, however, most of these play a role in cancer development and/or cell movement $[17,18]$. Furthermore, a number of growth factor/cytokine and metalloprotease genes are expressed in a THOC5 dependent manner $[17,18]$. We utilized tamoxifen inducible THOC5 knockout liver [31] for this experiment. We have previously shown that the depletion of THOC5 in liver did not result in pathological alteration. In addition, Albumin and Transferrin mRNAs are THOC5 independent [31]. As shown in Figure 5A, after tamoxifen treatment THOC5 was depleted within 4 days. In agreement with our previous data, although THOC5 is no longer detectable in the nucleus 4 days after injection, a pathological alteration was not observed in THOC5 depleted liver tissues (Figure 5B). We generated liver slices from THOC5 depleted and control liver (each 3-6 slices from 7 independent experiments) and then $1 \times 10^{5}$ GFP labeled MDA-MB-231 cells were incubated on each slice for 48 hours. We then quantified cancer cell adhesion to PCLS. 
A

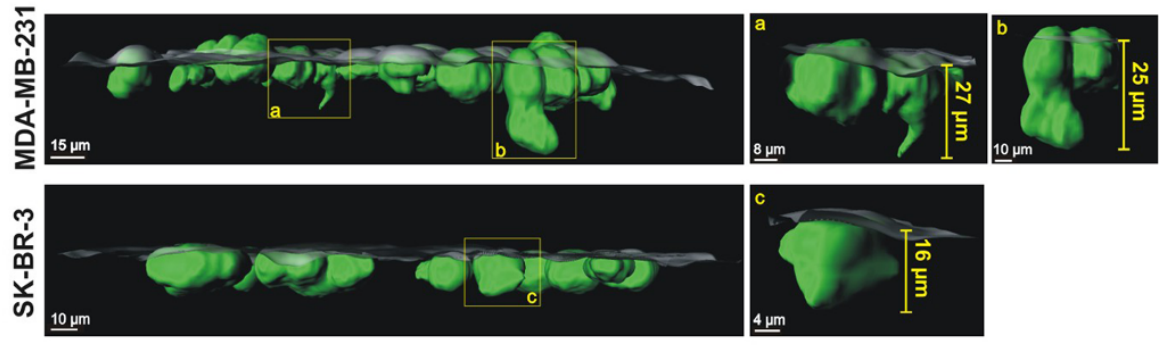

B

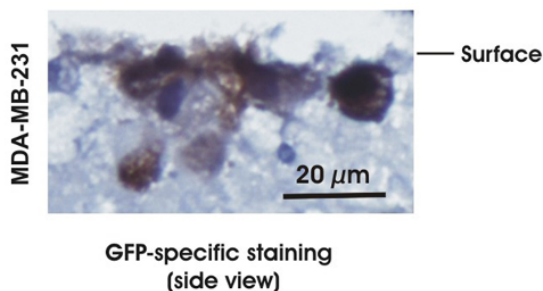

Figure 4 Application for observation of single cells in PCLS. (A): Partial penetration of MDA-MB-231 or SK-BR-3 cells (green) into PCLS (half transparent grey surface) was analyzed in a side view of IMARIS-generated 3-D images. Yellow frames indicate cancer cells that are extracted from the image to be shown separately $(a, b, c)$. (B): Paraffin sections of PCLS with MDA-MB-231 were stained by GFP specific antibody. Three independent experiments were performed and an example of representative data is shown here.

Strikingly, on the THOC5 KO liver slices with THOC5 expression levels depleted more than $50 \%$, numbers of adhered cancer cells were reduced to $17 \%$ of control liver slices (Figure 5C), suggesting that THOC5 KO liver lacks the adhesion potential for cancer cells. Our previous data obtained from transcriptome analysis using THOC5 depleted bone marrow macrophages, or mouse embryonic fibroblasts revealed that several chemokine/ growth factor genes or metalloprotease genes were THOC5 dependent $[17,18]$. To examine whether these genes were downregulated in the THOC5 KO PCLS, we performed quantitative RT-PCR using THOC5, Cxcl12, Pdgfa, Tgfb2, Tgfb3, Wnt11, and Mmpla genes specific primers (Table 1). Indeed these genes were downregulated more than 2-fold, while Albumin or GusB genes were not altered upon depletion of THOC5 (Figure 5D). These data imply that the PCLS ex vivo method can be applied to the study of tumor microenvironment and signal transduction at the molecular level.

\section{Discussion}

Cancer cells often break away from a primary tumor and travel through the blood and/or lymph system. These cancer cells do not always settle in and start new tumors, however. High rates of attrition are observed when cells exit the microvasculature into the parenchyma of tissue. For example, less than $3 \%$ of intravenously implanted cells survive to form micrometastases. Furthermore, the subsequent process of metastatic colonization is even more inefficient. Here, less than $0.02 \%$ of intravenously implanted cells generate macroscopic metastases [32]. It has been suggested that the genetic and/or epigenetic events drive the molecular evolution of these cells toward metastatic competence [25,33]. However exact molecular events in the interactions of cancer cells with tissueresident cells, micrometastasis formation followed by metastatic colonization are largely unknown.

Thus, the ability to analyze single tumor cell behavior in tissue as well as molecular events between tumor and organ resident cells is a major issue in the study of cancer metastasis. In an effort to achieve this goal we have established an ex vivo method using PCLS. We show that the viability of PCLS over time was improved when the slices were cultured in the presence of an oxygen carrier, PFD. It has been reported that PFD influences neurite outgrowth of retinal culture [34]. However, in this case cells were subjected to physical force from above, because PFD was placed on top of culture. PFD was applied as an oxygen supplier for 3-D culture of A431, BHK-21 and C2C12 cells [16]. It is not clear whether the application of PFD may influence functions of these cells. However, oxygen saturated PFD may support hepatocyte proliferation (regeneration) ex vivo. In the presence of PFD, we observed many mitotic hepatocytes in PCLS $24 \mathrm{~h}$ after slicing. Along the same line, in the presence of oxygen saturated PFD immediate early genes, such as $c-M y c, c-F o s$ or EGR-1 were upregulated 1 to $4 \mathrm{~h}$ after slicing (Tran and Tamura, unpublished data). 
A

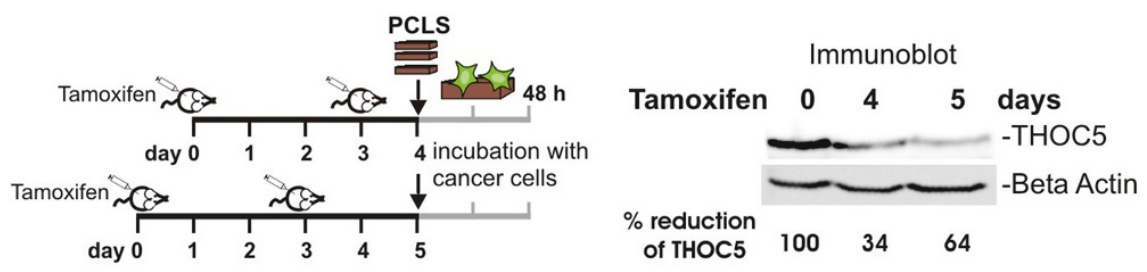

B
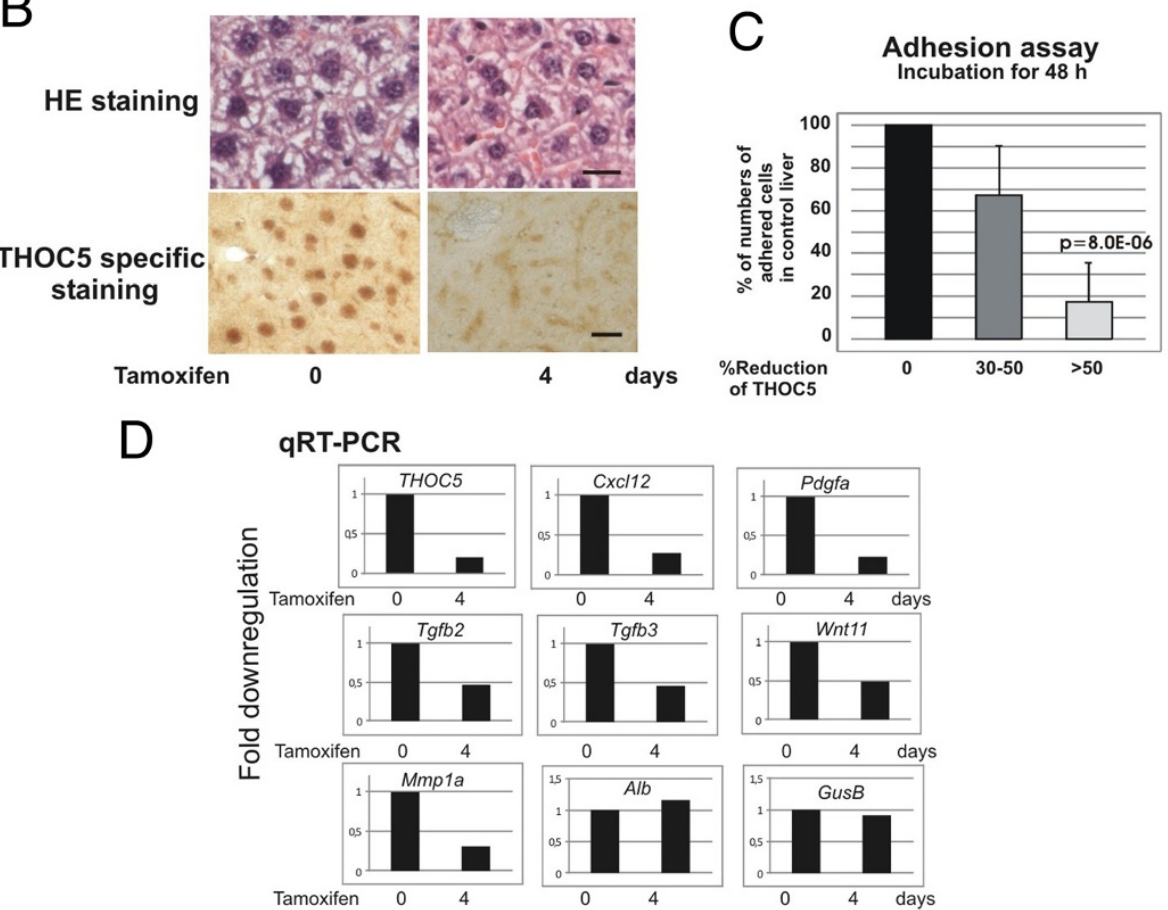

Figure 5 Tumor cell adhesion assay using PCLS derived from genetically manipulated THOC5 KO mice. (A): Rosa26-CreER ${ }^{\mathrm{T} 2}$ :THOC5 (flox/ flox) mice were injected with Tamoxifen as described previously [31]. Protein was extracted from organs on 0, 4 and 5 days after the first injection, and subjected to THOC5 and actin specific immunoblot. Total protein amount was standardized by actin (Beta Actin) specific immunoblot. Signal intensity of chemiluminescence was quantified using TINA 2.0 software (Raytest Isotopenmessgeraete GmbH, Straubenhardt, Germany) and percent (\%) reduction of THOC5 level is shown. (B): HE staining or THOC5 specific staining of paraffin sections from before (0 day) and after (4 days) tamoxifen injection. Bars represent $20 \mu \mathrm{m}$. Seven independent experiments were performed and an example of representative data is shown here. (C): GFP labelled MDA-MB-231 cells were plated onto PCLS from control liver or THOC5 depleted livers and incubated for $48 \mathrm{~h}$. GFP positive cells on the slices (percentage of number of adhered cells to numbers on the control slices) were counted from 3-6 slices of each samples from 7 THOC5 knockout mice. P value: student's t-test. (D): RNAs were extracted from liver and were supplied for the THOC5, CxCl12, Pdgfa, TGFb2, TGFb3, Wnt11, Mmp1a, Alb (Albumin), GusB, and Gapdh gene specific qRT-PCR (all primers are shown in Table 1). All data were standardized to the Gapdh level. Three independent experiments were performed.

It has been reported that liver regeneration induced by partial hepatectomy influences the growth of human colon cancer cells implanted into nude mice [35]. Thus, maintenance of the regenerative pathway by PFD may influence cancer cell adherence in the ex vivo system. At this time, however, the potential regenerative pathways in PCLS remain to be studied.

Using this system we demonstrated a difference in adhesion of two different human cancer cells (from two breast cancer cell lines) to mouse liver slices. This system may, therefore, also be useful in studying the association of cancer cells with liver-resident cells, including Kupffer cells and liver stromal cells that produce a number of chemotactic cytokines, growth factors and proteolytic enzymes [36-39], and in determining which cell types in the liver influence cancer cell invasion. Along the same lines, we further demonstrated that upon depletion of THOC5, a molecule that regulates approximately 100 genes, including chemotactic cytokines, growth factors and metalloprotease, tumor cells showed a reduced tendency to adhere to liver slices. Thus, improved PCLS methods may allow us to dissect signaling pathways on the molecular level using liver from genetically manipulated mice and cancer cells. 
Table 1 qRT-PCR primer pair sequences for selected genes

\begin{tabular}{|c|c|c|c|}
\hline Gene & Accession number & Forward primer & Reverse primer \\
\hline$A / b$ & NM_009654.3 & CAAGAGTGAGATCGCCCATCG & TTACTTCCTGCACTAATTTGGCA \\
\hline Cxcl12 & NM_013655.4 & TGCATCAGTGACGGTAAACCA & TTCTTCAGCCGTGCAACAATC \\
\hline Gapdh & NM_008084.2 & AGGTCGGTGTGAACGGATTTG & TGTAGACCATGTAGTTGAGGTCA \\
\hline GusB & NM_010368.1 & CCGACCTCTCGAACAACCG & GCTTCCCGTTCATACCACACC \\
\hline Mmp1a & NM_032006.3 & CCTTGATGAGACGTGGACCAA & ATGTGGTGTTGTTGCACCTGT \\
\hline Pdgfa & NM_008808.3 & CGATGAGGACCTGGGCTT & TTCTCGGGCACATGGTTAATG \\
\hline Tgfb2 & NM_009367.3 & AGAATCGTCCGCTITGATGTC & TCTGGTTTCACAACCTTGCT \\
\hline $\operatorname{Tgfb3}$ & NM_009368.3 & CCTATCAGGTCCTGGCACTTT & CTGCCCGGAACAGATTGGT \\
\hline THOC5 & NM_172438.3 & CTGTGTGCACTTCATGACTCTAAAGA & GAACTCCAGACATTTGGTGATCTCCT \\
\hline Wnt11 & NM_009519.2 & GGTGGTACACCGGCCTATG & TCACTGCCGTTGGAAGTCTTG \\
\hline
\end{tabular}

Alb: albumin (131 nt); Cxcl12: chemokine (C-X-C motif) ligand 12 (146 nt); Gapdh: glyceraldehyde-3 phosphate dehydrogenase (123 nt); GusB: glucuronidase, beta (169 nt); Mmp1a: matrix metallopeptidase 1a (238 nt); Pdgfa: platelet derived growth factor, alpha (232 nt); Tgfb2: transforming growth factor, beta 2 (198 nt); Tgfb3: transforming growth factor, beta 3 (252 nt); THOC5: THO complex subunit 5 homolog (178 nt), Wnt11: wingless-related MMTV integration site 11 (183 nt); (): product size.

We also demonstrated that single-cell behavior on the slices can be observed by confocal microscopy using the IMARIS image analysis program. Future applications may include tracing of the behavior of tumor cells as well as liver-resident cells at single-cell resolution by time lapse microscopy at the time that cancer cells establish first contact with the tissue slice. Furthermore, to study later stage of cancer cell infiltration in the liver, GFP-labelled cancer cells can be intrasplenically injected into mice, and then PCLS prepared at different time points for observing the invasive behavior of individual cancer cells using ex vivo system.

In addition, the use of experimental animals will be increasingly challenged in countries of the European Union, because of the adoption of new animal welfare rules in 2013. Although it is necessary to sacrifice one mouse, the liver from that one mouse can provide many slices, indicating that this ex vivo system can dramatically reduce the number of experimental animals required for this vital research.

Application of this method to cancer research is still in its earliest stages and it will be still necessary to develop better conditions to extend the life span of PCLS in the absence of growth factors and hormones. Furthermore, it will also be necessary to carefully compare the data from in vitro, in vivo and ex vivo studies. There can be no doubt, however, that this will become a useful method in the study of cancer.

\section{Conclusion}

Mouse PCLS culture in the presence of PFD may serve as a useful tool for observing the earlier invasion stages of malignant progression, such as the local adherence and invasiveness of individual cancer cells. This method may also prove useful for identification of factors in liver-resident cells that support cancer cell adherence and invasion using a gene knockout liver. Moreover, this method can partially supplant in vivo mouse experiments for prescreening anti-cancer reagents.

\section{Materials and methods}

\section{Preparation and incubation of PCLS}

As a source for PCLS whole livers were isolated from C57BL/6 mice or Wistar Rats. PCLS were prepared using a Krumdieck tissue slicer, MD6000 (Alabama Research and Development, Munford, AL, USA), filled with carbogen $\left(95 \% \mathrm{O}_{2} / 5 \% \mathrm{CO}_{2}\right)$ saturated ice-cold Krebs-Henseleit buffer $(5 \mathrm{mM} \mathrm{NaCl}, 118 \mathrm{mM} \mathrm{KCl}, 1.1 \mathrm{mM} \mathrm{MgSO}$, $1.2 \mathrm{mM} \mathrm{KH_{2 }} \mathrm{PO}_{4}, 25 \mathrm{mM} \mathrm{NaHCO} 3,2.5 \mathrm{mM} \mathrm{CaCl}$, $25 \mathrm{mM}$ D-Glucose, 9 mM HEPES, pH 7.42) [21]. Slices (25 mm ${ }^{2}$, slice thickness $\left.200-300 \mu \mathrm{m}\right)$ were transferred into Williams' Medium E (Gibco life technologies, Paisley, UK) supplemented with $14 \mathrm{mM}$ D-Glucose, $2.5 \mu \mathrm{g} / \mathrm{ml}$ Fungizone (Gibco life technologies), $294 \mu \mathrm{g} / \mathrm{ml} \mathrm{L-glutam-}$ ine, $60 \mu \mathrm{g} / \mathrm{ml}$ penicillin $\mathrm{G}$ and $50 \mu \mathrm{g} / \mathrm{ml}$ streptomycin) and were incubated at $37^{\circ} \mathrm{C}$ in an atmosphere with $5 \% \mathrm{CO}_{2}$ and $95 \%$ humidity. PFD was purchased by Sigma-Aldrich Chemie GmbH (München, Gemany).

\section{ATP assay and triglyceride colorimetric assay}

After incubation, slices were transferred in buffer containing $500 \mathrm{mM}$ Tris- $\mathrm{HCl}, \mathrm{pH} 7.8$ and $1 \mathrm{mM}$ EDTA, were frozen in liquid nitrogen and stored at $-80^{\circ} \mathrm{C}$. ATP was extracted from the slices by preparing homogenate in $0.5 \%$ TritonX-100 in $500 \mathrm{mM}$ Tris- $\mathrm{HCl}, \mathrm{pH} 7.8$ and $1 \mathrm{mM}$ EDTA and centrifugation for 5 minutes at 9,500 $\times \mathrm{g}$ at $4^{\circ} \mathrm{C}$. ATP level was measured in the sample using the ATP determination kit (Biaffin GmbH, Kassel, Germany). ATP values were normalized to the total protein content of the slice determined by Pierce $660 \mathrm{~nm}$ protein assay kit 
(Pierce Protein Biology Products, Rockford, IL, USA) using BSA for the calibration curve. Values displayed are relative value compared to the related controls. For triglyceride analysis, homogenates were prepared in the same buffer and measured by triglyceride colorimetric assay kit (Cayman Chemicals, Ann Arbor, MI, USA) according to the manufacturer's instructions.

\section{Cell culture and staining}

Human cancer cell line SK-BR-3 was grown in RPMI 1640 medium (Biochrom AG, Berlin, Germany) supplemented with $10 \%(\mathrm{v} / \mathrm{v})$ FCS. MDA-MB-231 cells were grown in Leibovitz's L15 medium (PAN Biotech, Aidenbach, Germany) supplemented with $10 \%$ (v/v) FCS. Cancer cells were infected with adenovirus carrying GFP (Ad-GFP, Vector Biolab, Philadelphia, PA, USA).

PCLS fixed by $3.7 \%$ formaldehyde were permeabilized by 0.2\% Triton-X 100 in PBS for 10 min at room temperature. Tissue was counterstained with $1.7 \mu \mathrm{M}$ TAMRA-SE (Life technologies, Carlsbad, CA, USA) for $2.5 \mathrm{~h}$ at $4^{\circ} \mathrm{C}$, washed once with $100 \mathrm{mM}$ glycine and twice with PBS before staining the nuclei using $0.5 \mu \mathrm{M}$ TO-PRO-3 iodide (Life technologies) for $1 \mathrm{~h}$ at $4^{\circ} \mathrm{C}$. After additional washing, PCLS were mounted on glass coverslips in Mowiol- solution containing DABCO.

\section{Confocal microscopy}

PCLS were examined using a confocal microscope LSM 510 Meta (Zeiss, Göttingen, Germany) 20× plan-neofluar objective and the $488 \mathrm{~nm}$ argon laser line, the $543 \mathrm{~nm}$ helium-neon 1 laser line and the $633 \mathrm{~nm}$ helium-neon 2 laser line for excitation, and emission filters BP 505-530, BP 560-615 and LP 650 for detection of GFP, TAMRA and TO-PRO-3 stain, respectively. Z-stacks were created with $2 \mu \mathrm{m}$ distance between the individual images. Z-stack image files were read into IMARIS 7.6.3 image analysis software (Bitplane scientific software, Zurich, Switzerland) and were converted into three dimensional representations. The surpass module of IMARIS was utilized to define the surface of PCLS and cancer cells from the channels recording TAMRA staining and GFP, respectively. 3-D representations of cancer cells in the tissue were visualized at different projection angles. Analysis of the depth of penetration of cancer cells into the PCLS was performed by defining the mean $\mathrm{z}$-coordinate of the tissue surface by measuring 20 points surrounding a cancer cell and determining the deepest point ( $\mathrm{z}$-coordinate) of the cancer cell.

\section{Immunoblotting procedures}

Details of immunoblotting have been described previously [40]. Mouse organs were extracted with lysis buffer containing $10 \mathrm{mM}$ Tris- $\mathrm{HCl}$ pH 7.6, $50 \mathrm{mM} \mathrm{NaF,} 1 \mathrm{mM}$ PMSF, $10 \mathrm{mM}$ EDTA, 1\% (w/v) Triton-X 100, 8 M Urea and protease inhibitor cocktail (Sigma, München, Germany). Polyclonal antibody against actin was purchased from Santa Cruz Biotechnology, Inc. (Santa Cruz, CA), and a monoclonal antibody against THOC5 was generated as described previously [41]. Corresponding proteins were visualized by incubation with peroxidase conjugated antimouse or anti-goat immunoglobulin followed by incubation with SuperSignal West FemtoMaximum Sensitivity Substrate (Pierce Protein Biology Products). Results were documented on a LAS4000 imaging system (GE Healthcare Bio-Sciences, Uppsala, Sweden).

\section{Immunohistochemistry}

Immunohistochemical studies were performed as detailed previously [42]. Polyclonal rabbit anti GFP antibody was from BD Biosciences (Heidelberg, Germany).

\section{Generation of THOC5 KO liver}

Generation of Rosa26-CreER ${ }^{\mathrm{T} 2}$ :THOC5 (flox/flox) mice has been described previously [31]. The deletion mutation of THOC5 was induced by intraperitoneal (i.p.) injection with tamoxifen twice at 3-day intervals. Four and five days after the first Tamoxifen injection mice were sacrificed and the livers were used in the preparation of PCLS.

\section{qRT-PCR analysis}

Twenty milligram samples of liver tissue were homogenized in cytoplasmic extraction buffer (Pierce Protein Biology Products Pierce). RNA was isolated from the whole tissue suspension with the High Pure RNA Isolation kit (Roche Diagnosis, Mannheim, Germany) according to the manufacturer's instructions. One microgram of RNA was reverse-transcribed using oligo dT primers and the Omniscript reverse transcriptase kit (Qiagen, Hilden, Germany) following the instructions provided. One-twentieth of the cDNA mix was used for real-time PCR using 10 pmol of forward and reverse primer and SensiFAST SYBR No-ROX kit (Bioline, London, UK) in Qiagen Rotor-Gene Q (Qiagen).

\section{Ethics statement}

Animal experiments were performed according to the German rules and regulations (Tierschutzgesetz), and approved by the ethics committee of Lower Saxony for care and use of laboratory animals: LAVES (Niedersächsisches Landesamt für Verbraucherschutz und Lebensmittelsicherheit). Mice were housed in the central animal facility of Hannover Medical School (ZTL) and were maintained as approved by the responsible Veterinary Officer of the City of Hannover. Animal welfare was supervised and approved by the Institutional Animal Welfare Officer (Tierschutzbeauftragter). 


\section{Abbreviations}

PCLS: Precision-cut liver slices; PFD: Perfluorodecalin; GFP: Green fluorescent protein; HPF: High-power field; HE: Hematoxylin and eosin.

\section{Competing interests}

The authors declare that they have no competing interests.

\section{Authors' contributions}

AK and ShS carried out PCLS culture, cancer cell labeling, invasion assay, ATP assay, confocal microscopy and data analysis. DDHT performed RT-PCR, and RK performed the pathological analyses, SKF, and KS supported preparation of the liver slices, and SuS performed IMARIS analysis. HT participated in the design of the study, and supervised the study. $A B$ participated in its design and coordination, and review of the manuscript. $T$ participated in the design of the study, contributed to the data analysis, and wrote and finalized the manuscript. All authors participated in the discussion and approved the final manuscript.

\section{Acknowledgements}

We thank C. Bruce Boschek for critically reading the manuscript, W. Müller for helpful discussion, and Martina Koch for supporting this project. A.K. and Sh.S. contributed equally to this work. This research was supported by DFG Ta-111/ 13-1, Deutsche Krebshilfe (111153), and PhD program Molecular Medicine and Leistungsorientierte Mittelvergabe with Frauenfaktor from $\mathrm{MHH}$.

\section{Author details}

${ }^{1}$ Institut fuer Biochemie, OE4310, Medizinische Hochschule Hannover, Carl-Neuberg-Str. 1, D-30623 Hannover, Germany. ${ }^{2}$ Fraunhofer Institut für Toxikologie und Experimentelle Medizin Atemwegspharmakologie, Nikolai-Fuchs-Str.1, D-30625 Hannover, Germany. ${ }^{3}$ Institute of Veterinary Pathology, Freie Universitaet Berlin, Robert-von-Ostertag- Str. 15, D-14163 Berlin, Germany.

Received: 5 September 2014 Accepted: 23 October 2014

Published online: 07 November 2014

\section{References}

1. Qian F, Vaux DL, Weissman IL: Expression of the integrin alpha 4 beta 1 on melanoma cells can inhibit the invasive stage of metastasis formation. Cell 1994, 77(3):335-347.

2. McClatchey Al: Modeling metastasis in the mouse. Oncogene 1999, 18(38):5334-5339.

3. Boess F, Kamber M, Romer S, Gasser R, Muller D, Albertini S, Suter L: Gene expression in two hepatic cell lines, cultured primary hepatocytes, and liver slices compared to the in vivo liver gene expression in rats: possible implications for toxicogenomics use of in vitro systems. Toxicol Sci 2003, 73(2):386-402.

4. Klassen LW, Thiele GM, Duryee MJ, Schaffert CS, DeVeney AL, Hunter CD, Olinga P, Tuma DJ: An in vitro method of alcoholic liver injury using precision-cut liver slices from rats. Biochem Pharmacol 2008, 76(3):426-436.

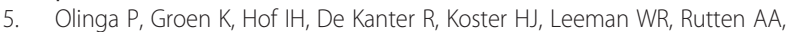
Van Twillert K, Groothuis GM: Comparison of five incubation systems for rat liver slices using functional and viability parameters. J Pharmacol Toxicol Methods 1997, 38(2):59-69.

6. Muller D, Glockner R, Rost M, Steinmetzer P: Monooxygenation, cytochrome P450-mRNA expression and other functions in precision-cut rat liver slices. Exp Toxicol Pathol 1998, 50(4-6):507-513.

7. Glockner R, Steinmetzer P, Drobner C, Muller D: Use of fresh and cryopreserved human liver slices in toxicology with special reference to in vitro induction of cytochrome P450. Toxicol In Vitro 1999, 13(4-5):531-535

8. Graaf IA, Groothuis GM, Olinga P: Precision-cut tissue slices as a tool to predict metabolism of novel drugs. Expert Opin Drug Metab Toxicol 2007, 3(6):879-898.

9. Van de Bovenkamp M, Groothuis GM, Meijer DK, Olinga P: Liver slices as a model to study fibrogenesis and test the effects of anti-fibrotic drugs on fibrogenic cells in human liver. Toxicol In Vitro 2008, 22(3):771-778.

10. Olinga P, Schuppan D: Precision-cut liver slices: a tool to model the liver ex vivo. J Hepatol 2013, 58(6):1252-1253.

11. Riess JG: Oxygen carriers ("blood substitutes")-raison d'etre, chemistry, and some physiology. Chem Rev 2001, 101(9):2797-2920.
12. Lowe KC: Engineering blood: synthetic substitutes from fluorinated compounds. Tissue Eng 2003, 9(3):389-399.

13. Lowe KC: Blood substitutes: from chemstry to clinic. J Mater Chem 2006, 16:4189-4196.

14. Juszczak MT, Elsadig A, Kumar A, Muzyamba M, Pawelec K, Powis SH, Press M: Use of perfluorodecalin for pancreatic islet culture prior to transplantation: a liquid-liquid interface culture system-preliminary report. Cell Transplant 2011, 20(2):323-332.

15. Lowe KC: Perfluorochemical respiratory gas carriers: benefits to cell culture systems. J Fluorine Chem 2002, 118:19-26.

16. Pilarek M, Grabowska I, Ciemerych MA, Dabkowska K, Szewczyk KW: Morphology and growth of mammalian cells in a liquid/liquid culture system supported with oxygenated perfluorodecalin. Biotechnol Lett 2013, 35(9):1387-1394

17. Guria A, Tran DD, Ramachandran S, Koch A, El Bounkari O, Dutta P, Hauser $H$, Tamura T: Identification of mRNAs that are spliced but not exported to the cytoplasm in the absence of THOC5 in mouse embryo fibroblasts. RNA (New York NY) 2011, 17:1048-1056.

18. Tran DD, Saran S, Dittrich-Breiholz O, Williamson AJ, Klebba-Farber S, Koch $A$, Kracht $M$, Whetton AD, Tamura T: Transcriptional regulation of immediate-early gene response by THOC5, a member of mRNA export complex, contributes to the M-CSF-induced macrophage differentiation. Cell Death Dis 2013, 4:e879.

19. Westra IM, Oosterhuis D, Groothuis GM, Olinga P: The effect of antifibrotic drugs in rat precision-cut fibrotic liver slices. PLoS One 2014, 9(4):e95462.

20. Westra IM, Oosterhuis D, Groothuis GM, Olinga P: Precision-cut liver slices as a model for the early onset of liver fibrosis to test antifibrotic drugs. Toxicol Appl Pharmacol 2014, 274(2):328-338

21. de Graaf IA, Olinga P, de Jager MH, Merema MT, de Kanter $R$, van de Kerkhof EG, Groothuis GM: Preparation and incubation of precision-cut liver and intestinal slices for application in drug metabolism and toxicity studies. Nat Protoc 2010, 5(9):1540-1551.

22. Vickers AE, Saulnier M, Cruz E, Merema MT, Rose K, Bentley P, Olinga P: Organ slice viability extended for pathway characterization: an in vitro model to investigate fibrosis. Toxicol Sci 2004, 82(2):534-544

23. Olinga $\mathrm{P}$, Hof $\mathrm{IH}$, Merema MT, Smit M, de Jager MH, Swart PJ, Slooff MJ, Meijer DK, Groothuis GM: The applicability of rat and human liver slices to the study of mechanisms of hepatic drug uptake. J Pharmacol Toxicol Methods 2001, 45(1):55-63.

24. Beckwith $H$, Yee D: Insulin-like growth factors, insulin, and growth hormone signaling in breast cancer: implications for targeted therapy. Endocr Pract 2014, 1-18 [Epub ahead of print]

25. Valastyan S, Weinberg RA: Tumor metastasis: molecular insights and evolving paradigms. Cell 2011, 147(2):275-292.

26. Thompson EW, Paik S, Brunner N, Sommers CL, Zugmaier G, Clarke R, Shima TB, Torri J, Donahue S, Lippman ME, Martin GR, Dickson RB: Association of increased basement membrane invasiveness with absence of estrogen receptor and expression of vimentin in human breast cancer cell lines. J Cell Physiol 1992, 150(3):534-544.

27. Morini M, Mottolese M, Ferrari N, Ghiorzo F, Buglioni S, Mortarini R, Noonan DM, Natali PG, Albini A: The alpha 3 beta 1 integrin is associated with mammary carcinoma cell metastasis, invasion, and gelatinase B (MMP-9) activity. Int J Cancer 2000, 87(3):336-342.

28. Paulo CS, Lino MM, Matos AA, Ferreira LS: Differential internalization of amphotericin B-conjugated nanoparticles in human cells and the expression of heat shock protein 70. Biomaterials 2013, 34(21):5281-5293.

29. Paz H, Pathak N, Yang J: Invading one step at a time: the role of invadopodia in tumor metastasis. Oncogene 2013, 33(33):4193-4202.

30. Tran DD, Koch A, Tamura T: THOC5, a member of the mRNA export complex: a novel link between mRNA export machinery and signal transduction pathways in cell proliferation and differentiation. Cell Commun Signal 2014, 12:3.

31. Saran S, Tran DD, Klebba-Farber S, Moran-Losada P, Wiehlmann L, Koch A, Chopra H, Pabst O, Hoffmann A, Klopfleisch R, Tamura T: THOC5, a member of the mRNA export complex, contributes to processing of a subset of wingless/integrated (Wnt) target mRNAs and integrity of the gut epithelial barrier. BMC Cell Biol 2013, 14(1):51.

32. Luzzi KJ, MacDonald IC, Schmidt EE, Kerkvliet N, Morris VL, Chambers AF, Groom AC: Multistep nature of metastatic inefficiency: dormancy of solitary cells after successful extravasation and limited survival of early micrometastases. Am J Pathol 1998, 153(3):865-873. 
33. Fidler IJ: The pathogenesis of cancer metastasis: the 'seed and soil' hypothesis revisited. Nat Rev 2003, 3(6):453-458.

34. Malchiodi-Albedi F, Matteucci A, Formisano G, Paradisi S, Carnovale-Scalzo G, Perilli R, Scorcia G, Caiazza S: Perfluorohexyloctane (F6H8) induces structural modifications and increases apoptosis in rat primary retinal cultures. J Biomed Mater Res B Appl Biomater 2003, 65(1):133-136.

35. Gutman M, Singh RK, Price JE, Fan D, Fidler IJ: Accelerated growth of human colon cancer cells in nude mice undergoing liver regeneration. Invasion Metastasis 1994, 14(1-6):362-371.

36. Paschos KA, Majeed AW, Bird NC: Role of Kupffer cells in the outgrowth of colorectal cancer liver metastases. Hepatol Res 2010, 40(1):83-94.

37. Bayon LG, Izquierdo MA, Sirovich I, van Rooijen N, Beelen RH, Meijer S: Role of Kupffer cells in arresting circulating tumor cells and controlling metastatic growth in the liver. Hepatology (Baltimore, Md) 1996, 23(5):1224-1231.

38. Bilzer M, Roggel F, Gerbes AL: Role of Kupffer cells in host defense and liver disease. Liver Int 2006, 26(10):1175-1186.

39. Gout S, Huot J: Role of cancer microenvironment in metastasis: focus on colon cancer. Cancer Microenviron 2008, 1(1):69-83.

40. Koch A, Scherr M, Breyer B, Mancini A, Kardinal C, Battmer K, Eder M, Tamura T: Inhibition of Abl tyrosine kinase enhances nerve growth factor-mediated signaling in Bcr-Abl transformed cells via the alteration of signaling complex and the receptor turnover. Oncogene 2008, 27(34):4678-4689.

41. Mancini A, El Bounkari O, Norrenbrock AF, Scherr M, Schaefer D, Eder M, Banham AH, Pulford K, Lyne L, Whetton AD, Tamura T: FMIP controls the adipocyte lineage commitment of $\mathrm{C} 2 \mathrm{C} 12$ cells by downmodulation of C/EBP alpha. Oncogene 2007, 26(7):1020-1027.

42. Klopfleisch R, Von Deetzen M, Weiss AT, Weigner J, Weigner F, Plendl J, Gruber AD: Weigners fixative-an alternative to formalin fixation for histology with improved preservation of nucleic acids. Vet Pathol 2012, 50(1):191-199

doi:10.1186/s12964-014-0073-7

Cite this article as: Koch et al:: Murine precision-cut liver slices (PCLS): a new tool for studying tumor microenvironments and cell signaling ex vivo. Cell Communication and Signaling 2014 12:73.

\section{Submit your next manuscript to BioMed Central and take full advantage of:}

- Convenient online submission

- Thorough peer review

- No space constraints or color figure charges

- Immediate publication on acceptance

- Inclusion in PubMed, CAS, Scopus and Google Scholar

- Research which is freely available for redistribution 